\title{
A neurobiologia da psicopatologia e psicoterapia e as implicações práticas de uma perspectiva materialista na definição de mente*
}

\section{Neurobiology of Psychopathology and Psychotherapy and practical implications of materialistic view in mind definition} inclications of materialiss

DOI: $10.11144 / J a v e r i a n a . u p s y 15-5 . n p p i$

Recepção: 20 Maio 2016 | Aprovação: 13 Dezembro 2016

\author{
Rodrigo TeIXeIra Lopes ${ }^{\mathrm{a}}$ \\ U niversidade C atólica de Petrópolis, Brasil \\ Marcele de Carvalho \\ Universidade Federal do Rio de Janeiro, Brasil \\ Felipe Santos de Oliveira \\ Universidade Católica de Petrópolis, Brasil \\ Óscar F. Gonçal ves b \\ Universidade do M inho, Portugal
}

\footnotetext{
aA correspondência sobre esse artigo deve ser toda dirigida para o priemeiro autor. E-mail: rodrigo.lopes@ucp.br

${ }^{\mathrm{b}} \mathrm{N}$ este artigo os autores retomam as ideias centrais apresentadas no curso A profundamentos em Psicopatologia, ministrado pelo autor sênior (Prof. O scar Gonçalves).
}

Para citar este artículo: Lopes, R., De Carvalho, M. R. Oliveira, F. S., \& Gonçalves, Ó. F. (2016) A neurobiologia da psicopatologia e psicoterapia e as implicações práticas de uma perspectiva materialista na definição de mente. Universitas Psychologica, 15 http://dx.doi.org/10.11144/Javeriana.upsy15-5.nppi

\section{RESUMO}

A psicologia passa no momento atual por uma importante mudança de paradigma teórico e conceitual. Essa mudança ficou bastante marcada na década de 90, considerada a "década do cérebro", quando aumentaram significativamente o número de estudos em comportamento que baseavam-se no funcionamento do cérebro. $O$ mainstream das ciências do comportamento retornou à noção monista materialista de que cérebro e comportamento estão intrinsecamente interligados. Essa noção saiu dos laboratórios de pesquisa básica e chegou com força nas pesquisas aplicadas à psicopatologia e à psicoterapia. A presente revisão narrativa tem como principal objetivo traçar um panorama atual desse empreendimento científico, com ênfase nos esforços feitos dentro das áreas da psicopatologia e da psicologia clínica. Ao final, discute-se até que ponto essa mudança de paradigma pode chegar na prática profissional do psicólogo.

\section{Palavras-chave}

psicologia clínica; psicopatologia; neurociências; psiquiatria; neurologia; marcadores biológicos.

\section{ABSTRACT}

Psychology faces nowadays for a major change of theoretical and conceptual paradigm. This change was quite marked in the 90s, which was considered the "decade of the brain" when the number of behavioral studies based on brain functioning significantly increased. The mainstream of the behavioral sciences returned to the monistic materialistic notion that the brain and behavior are inextricably intertwined. This idea came out of basic research laboratories and influenced strongly in applied research in psychopathology and psychotherapy. This narrative review aims to draw up a current view of the scientific enterprise, with emphasis on efforts within the areas of clinical psychology and psychopathology. It is also discussed to what extent this paradigm shift can reach the professional practice of psychology.

Keywords 
clinical psychology; psychopathology; neuroscience; psychiatry; neurology; biological markers.

\section{Introdução}

O problema filosófico da relação corpo-alma, atualmente referido como problema mentecérebro, é uma das questões centrais dentro da filosofia da mente (McGinn, 1996). O dualismo cartesiano constituiu-se como uma proposta de resolução do problema quando postula a existência de duas substâncias distintas: a res cogitans e a res extensa (Churchland, 1993). Para Descartes, o homem é formado por duas naturezas distintas que são capazes de se interinfluenciarem. A primeira substância seria não-física, não-divisível e subjetiva, a qual ele chama de Espírito. A segunda seria o corpo tal como podemos percebê-lo no mundo físico, sujeito às leis da física. Descartes não se preocupou muito em explicar a interação entre essas duas substâncias e bastou-lhe dizer que ela se dava através de uma pequena glândula no cérebro, a glândula pineal (Churchland, 1993; McGinn, 1996).

No âmbito das ciências do comportamento, isso não foi assim tão diferente (Araújo, 2003). A psicologia, ao se constituir como uma disciplina científica independente da filosofia teve como objeto de estudo os "processos mentais", diferente da medicina e da biologia, que estudavam o corpo. Por mais que mudassem os nomes desses "processos mentais", os psicólogos, durante muito tempo, se apoiaram na noção de que mente e corpo eram duas coisas distintas, tal como postulou o filósofo francês. Consequências desse dualismo foram a falta de rigor científico na psicoterapia e a emergência e declínio de inúmeros modelos terapêuticos ao longo da história (Gonçalves et al., 2006; Kandel, 1998).

A década de 90, conhecida como a biólogos moleculares, geneticistas, neurocirurgiões, neurocientistas computacionais, psiconeuroimunologistas e, a partir da observação clínica, de psiquiatras, neurologistas e psicólogos, ajudaram a desvendar a "glândula pineal" de Descartes. A psicopatologia seguiu essa direção utilizando a grande variedade de novas técnicas científicas para estudar mais a fundo as relações entre cérebro, transtorno mental e tratamento. O princípio norteador dessa tendência, a qual os filósofos da mente denominam "materialismo" (Churchland, 1993), é de que os processos psicológicos (e.g., percepção, atenção, memória, linguagem, funcionamento executivo, emoções, aprendizagem), inclusive os patológicos, ao mesmo tempo que derivam de mecanismos neurobiológicos, também desencadeiam alterações neurobiológicas (Eisch \& Nestler, 2002; Gonçalves et al., 2006; Kandel, 1998).

A presente revisão narrativa pretende, primeiramente, traçar um panorama dos avanços feitos na psicopatologia e seu tratamento a partir dessa visão "materialista" e, consequentemente, cientificista do ser humano. Posteriormente, serão discutidas algumas consequências práticas que podem derivar dessa perspectiva.

\section{A psicopatologia e a psicoterapia com bases biológicas e científicas}

Kandel (1998) constatou que a psiquiatria (e o mesmo vale para a psicologia clínica), graças à forte influência da psicanálise, permaneceu por muito tempo como uma ciência empírica, com pobres bases científicas. $\mathrm{O}$ autor enfatizou a necessidade por mais estudos que tentassem com preender a psicopatologia DWDYpVOTIXV bases neurobiológicas. 㘞

Uma primeira grande questão que se faz

"década do cérebro", marca uma mudanca

m. min.m.

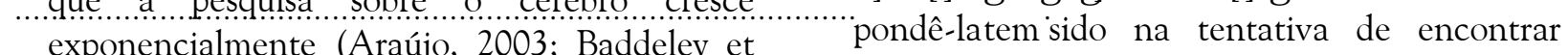
m. m. 


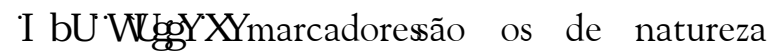
bioquímica, os quais apresentar-se-á logo em seguida. Posteriormente, serão descritos estudos que evidenciam características estruturais e funcionais do cérebro que estão na base de diferentes psicopatologias.

\section{A base neuroquímica para a psicopatologia e psicoterapia}

Não são poucas as evidências de mudanças significativas em níveis bioquímicos durante intervenções psicológicas. ( $\mathrm{P}[\mathrm{Xm} \square$ tudo $\mathbb{F}$ om $\mathbb{W}$ adolescentes do sexo feminino voluntárias, com idade média de 16 anos, que preencheram os critérios diagnósticos para depressão leve, Jeong et al. (2005) encontraram[ho grupo experimental submetido à intervenção com Terapia de Dança e Movimento, um aumento significativo na concentração da serotonina plasmática e um declínio da dopamina, indicadores que estão

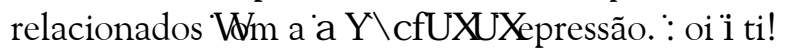
lizadođambém o Symptoms CheckList-90 (De— rrogatis,[1977)[\$ara[P edir RQRvel[Ge \$Serturbação psicológica, a qual variou na mesma proporção da medida biológica.

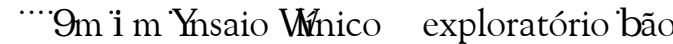
randomizado com 20 pacientes hospitalizados, diagnosticados com T UOQMRLOR] HUHXOR

Malor (escore mínimo de 18 na Hamilton Depression Scale, HAMD) $\square$ e não medicados com antidepressivos, obtiveram resultados que confirmaram a hipótese de que a fosforilação do CREB aumenta depois de duas semanas de tratamentos antidepressivos de quaisquer espécie. Participaram da pesquisa pacientes que estavam recebendo tratamento exclusivamente psicológico e exclusivamente psicofarmacológico. Dos 15 UHSRQGQWMALRD WDAP HQR IIfforam tratados somente com psicoterapia (4 comtterapia interpessoal e 1 não especificado). Foiffeita uma coleta de sangue no primeiro, sétimo $\$$ décimo quarto dia de trata $\square$ mento. A resposta dlo tratamento foi definida como pelo menos $\$ 0 \%$ de redução no escore inicial da HAMD五a segunda coleta de sangue. O objetivo desse@studo foi verificar se a fosfori $\square$ lação do CREB nos[linfócitos $T$ do sangue peri! férico poderia servir łomo um marcador de es $\square$ tado da resposta dotratamento. Houveram diferenças significativas@entre os 15 respondentes e os 5 não respondentes Go tratamento no que tange à fosforilação dođREB.

Jockers-Scherübl et al. (2007) mediram as concentrações de serum de Nerve Growth Factor (NGF) em 22 pacientes com o diagnóstico de Transtorno de Ansiedade Generalizada (TAG) $\square$ antes e depois do tratamento com Terapia Cognitivo-Comportamental (TCC) $\square$ e compararam com um grupo controlHde sujeitos considerados saudáveis. Antes de iniciar a terapia os sujeitos de ambos os grupos não mostraram diferenças. No final da TCC bem sucedida os níveis de concentração de serum de NGF dos pacientes com TAG aumentou significativamente comparativamente com o grupo controlH $\mathrm{O}$ aumento significativo dos níveis de NGF depois de 25 sessões de tratamento com TCC UHOWXXmađnudança neurobiológica significativa e positiva, ilndicando uma boa resposta terapêutica. Entre $\phi s \quad 22 \mathrm{su} \square$ jeitos da amostra, 3 não responderam ło trata $\square$ mento e não apresentaram aumento ignifica

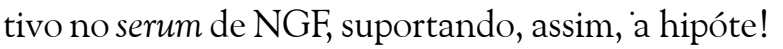
se de que o aumento da concentração de serum de NGF pode ser um indicador de maior sucesso no tratamento de TAG com TCC.

O hormônio DAYQRFRLWFRWy/LFRTACTH) HD

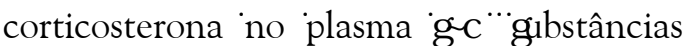
associadas ao estresse agudo. Na tentativa de encontrar os mecanismos biológicos subjacentes aos cuidados maternais, Meaney \& Szyf (2005) demostraram que a descendência das mães adultas que manifestam elevados cuidados maternais após a semana do parto apresentRXXm[Eaixo nível de ACTH, quando comparada com a descendência de mães adultas com poucos cuidados maternos.

Até agora, vimos alguns estudos com uma metodologia "top down", estudoV que nos permitem perceber "de cima para baixo" como intervenções comportamentais provocam alterações neuroquímicas significativas: uma 
melhora dos sintomas depressivos aumenta a concentração da serotonina plasmática e diminui a concentração da dopamina (Jeong et al., 2005), aumenta a fosforilação do CREB (Koch et al., 2002) —a melhora nos sintomas de TAG aumenta a concentração no serum de NGF (Jockers-

Scherübl et al., 2007); um maior cuidado maternal diminui a concentração de ACTH.

Outro tipo de investigação evidencia as mudanças de baixo para cima ("bottom up"). Um exemplo disso é o estudo experimental duplo-cego que se segue. Apesar de não ter sido desenhado para nenhuma psicopatologia específica, mostra resultados que ajudarão muito no tratamento do autismo. Domes, Heinrichs, Michel, Berger, \& Herpertz (2007) pediram a 31 voluntários masculinos saudáveis que inalassem uma certa quantidade de oxitocina. Para alguns deles foi dado placebo. Tal hormônio está muito envolvido em processos de vinculação e, até por isso, presente em maiores concentrações em mulheres grávidas. Foi pedido que os participantes fizessem um teste que avalia a capacidade de inferir estados mentais em outras pessoas através da expressão de seus olhos, o "Reading the mind in the eyes test" (RMET). Os resultados mostraram que houve uma alteração muito significativa nos escores do RMET entre os sujeitos que inalaram oxitocina e aqueles que inalaram placebo. Percebemos aqui uma alteração comportamental clara, em decorrência de uma manipulação biológica.

A neurogênese como base para compreender a psicopatologia e psicoterapia

Uma outra linha de investigação importante baseada na biologia para a compreensão da psicopatologia deriva dos estudos sobre a neurogênese. Até pouco tempo atrás, pensavase que a neurogênese não existia nos adultos. Em uma completa revisão sobre o tema, Eisch \& Nestler (2002) apontalDn @ relação[htre $\mathbb{D}$ neurogênese e alguns transtornos psiquiátricos. Os autores defendel $\mathbf{m}$ [Tue a Qurogênese relacionada com a psicopatologia, não somente por afetar a morfologia e o volume das estruturas, o que também acontece, mas também porque a neuro e a citogênese estão diretamente ligadas à plasticidade neuronal. Evidências disso, segundo os autores, advém do fato de que a neurogênese no adulto ocorre em zonas com alto grau de plasticidade neuronal, tais como o hipocampo e o bulbo olfatório. Além disso, tem sido encontrada neurogênese no adulto em todos os mamíferos estudados. Uma outra evidência é que a neurogênese é regulada por estímulos ambientais e fisiológicos.

Uma importante conclusão desse estudo é a de que a neurogênese está muito relacionada à regulação do hipocampo, e é aqui que está a sua grande associação com a psicopatologia. Em casos de estresse crônico e de estresse póstraumático, o volume do hipocampo é bem mais reduzido, se comparado com controles. A resposta ao estresse, de maneira prolongada, é apontada como um fator importante na etiologia da depressão (Scorza, Guerra, Cavalheiro, \& Calil, 2005). Sheline, Wang, Gado, Csernansky, \& Vannier (1996) realizaram estudos de neuroimagm que mostraram uma diminuição do volume hipocampal de mulheres idosas com depressão quando comparadas com mulheres sem patologia associada e da mesma faixa etária. Os autores associaram essa perda neuronal a uma neurotoxicidade mediada pelo estresse. $\mathrm{O}$ estresse agudo ou crônico diminui a proliferação e sobrevivência dos neurônios recentemente nascidos nas zonas subgranulares adultas em várias espécies de mamíferos, muito provavelmente através da mediação de glicocorticóides, com a consequente diminuição total do volume do hipocampo e de prejuízos de memória (Fuchs, Uno, \& Flügge, 1995).

Da mesma forma que a psicopatologia altera o processo de neurogênese, a introdução de mudanças ambientais e comportamentais tais como o exercício voluntário, a exposição à ambientes ricos em estímulos e, claro, a psicoterapia, também o faz. Entender melhor a neurogênese e conseguir manipulá-la é conseguir também aumentar a eficácia de tratamentos em saúde mental, inclusive a eficácia de técnicas psicológicas específicas (Scorza et al., 2005). 
A sinaptogênese como base para a mudança em psicoterapia

Apesar da importância da neurogênese em processos psicológicos e psicopatológicos, tal como vimos acima, é na sinaptogênese que reside o maior número de evidências para a compreensão da relação entre cérebro e ambiente. Isso se dá porque a neurogênese é quantitativa e qualitativamente mais limitada do que a sinaptogênese. Quantitativamente, pois a primeira acontece em proporções muito pequenas no humano adulto, se comparada com sinaptogênese. Qualitativamente porque a sinaptogênese é claramente um processo funcional e mais diretamente dependente da adaptação do organismo às condições externas a ele, o que a torna também mais dinâmica. É a sinaptogênese que está mais diretamente envolvida com os processos de plasticidade, o que a torna o "calcanhar de Aquiles do cérebro" (Johnston, 2004). -RKQXRQ WDODण nos a adaptar em nosso meio é também uma condição fundamental para o desenvolvimento da psicopatologia.

As técnicas de QHXRP DHP, tais [Fomo Tomografia de Emissão de Positrões (PET) e Ressonância Magnética Funcional (fMRI) abriram as portas para uma exploração não invasiva do cérebro humano, muitas vezes em um nível de resolução que possibilita vermos as mudanças ao nível estrutural e funcional associadas com os sintomas dos trastornos e com a evolução do tratamento (Kumari, 2006).

Do ponto de vista do desenvolvimento cognitivo temos, ha uma linha de estudos que mostra como a plasticidade está envolvida nos processos de mudança. Ochsner, Bunge, Gross, \& Gabrieli, (2002) observaram a relação entre a reavaliação do estímulo negativo ("reappraisal", um mecanismo importantíssimo para a psicoterapia), a melhoria do humor e os padrões de atividade cerebral. Os sujeitos foram avaliados antes e depois de lhes ser pedido para reavaliar (ou reinterpretar) cenas altamente negativas sob uma luz mais positiva.
A reavaliação foi associada com a melhoria do humor e aumento da atividade do córtex pré-

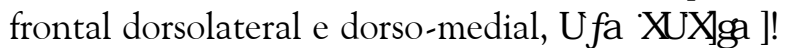

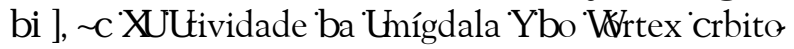
frontal.

No nível psicopatológico, encontramos algumas evidências já bastante estabelecidas de processos e áreas cerebrais responsáveis por estados psicopatológicos. A depressão, por exemplo, está comumente associada com áreas dorsais, inclusive o córtex pré-frontal dorsolateral (Roffman, Marci, Glick, Dougherty, \& Rauch, 2005). Goldapple et al. (2004) RENHYDDP

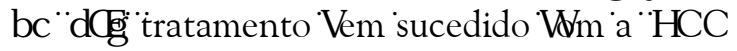
que a ativação de múltiplas áreas frontais e parietais diminuiu e que houve um aumento no metabolismo do hipocampo. Na revisão feita por Kumari (2006) notou-se ainda que, depois de alguns tratamentos psicoterapêuticos bem sucedidos houve um aumento de fluxo sanguíneo nos gânglios basais direitos.

Nas fobias o aumento de fluxo sanguíneo no córtex visual associativo e diminuição do fluxo sanguíneo no hipocampo, cingulado posterior, córtex orbito-frontal, pré-frontal e temporal, encontram-se associados ao medo e ansiedade que emergem devidom向四stimulação四óbica.

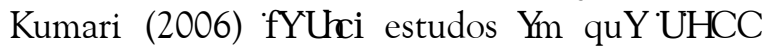
de sucesso modifica a atividade neuronal no córtex pré-frontal dorsolateral (o que reflete o uso de estratégias meta-cognitivas proativas com o objetivo de autorregular o medo e ansiedade) e da circunvolução para-hipocampal (relacionada com a automática reativação da memória do medo). Já na esquizofrenia $\square$ as regiões cerebrais afetadas são o córtex frontal, temporal e estriado, a amigdala, o hipocampo e o tálamo. A TCC tem demonstrado eficácia na diminuição dos sintomas positivos da esquizofrenia e grande influência nos sintomas negativos. Contudo, não existe nenhum artigo publicado no que respeita à correlação das bases neuronais e os efeitos da Terapia Cognitivo-Comportamental na esquizofrenia (Kumari, 2006).

No Transtorno Obsessivo-CompulsivR (TOC), Nakatani et al. (2003) encontraram disfunções no córtex orbito-frontal, no córtex do cingulado e no núcleo caudado em pacientes 
com esse transtorno. Depois de submetidos à terapia comportamental, baseada em exposição e prevenção de resposta, notou-se uma redução significativa da taxa de metabolismo no núcleo caudado direito nos respondentes. Esses resultados foram consistentes com aqueles encontrados em outras revisões sobre o tema (Kumari, 2006; Roffman et al., 2005).

É interessante notar que no caso do

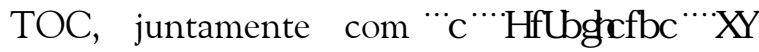
Pânicoநos lesultados encontrados \tăo blastante semelhantes, tanto para os tratamentos psicoterápicos, quanto para os farmacológicos (Kumari, 2006), o que serve como mais uma evidência para a hipótese de que toda intervenção psicológica é também uma intervenção cerebral, seja no nível bioquímico, celular ou cerebral.

Discussão: implicações práticas para o psicólogo clínico

Em 2007, o então atual presidente da American Psychological Association (APA), fH um chamado veemente aos investigadores dos processos de mudança em psicoterapia para que focalizDKem os seus próximos esforços em achar RVtrnecanismos e mediadoreVldsponsáveis \$Sara a eficácia da terapia (Kazdin, 2007). Já se sabe que a psicoterapia ajuda, mas RVP IFDQVP RVQRRVhR DQGDFRQKHFLRV Wesar das lnúmeras tentativas de responder a essa questão (Gonçalves et al., 2006). Como vimos acima, os investigadores das neurociências cognitivas são responsáveis pela talvez mais importante linha de investigação

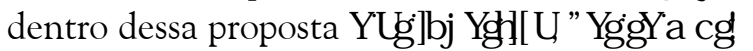
WDP [promissores.

Tal como previa Kandel (1998), assistimos hoje no plano científico, quase dez anos depois, à uma cooperação cada vez maior entre a neurologia, psiquiatria e a psicologia clínica e um crescente entusiasmo com o modelo biológico na saúde mental. No plano profissional da psicologia, isso é sentido pela emergência da neuropsicologia Łenquanto uma prática instituída e muito reconhecida dentre as profissões da saúde.
Uma perspectiva para o futuro é que à

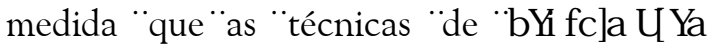
forem DSHUHoRDCDWestas Sodem ser - teis não apenas para efetuar o diagnóstico de várias doenças mentais mas também para monitorizar o progresso da intervenção psicológica, o que nos tiraria do presente estado de "tentativa e erro" em que se encontra a psicoterapia (Roffman et al., 2005). Isso poderá ser um grande avanço para a área, no sentido de uma maior potencialidade em saber o que $\mathbb{p}$

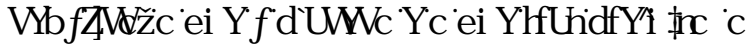
que faria com que DHIFi FWIGRVIWDIP HQR

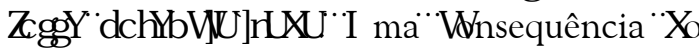
maior rigor científico na psicoterapia seria o maior reconhecimento por parte do grande público e das outras profissões de saúde,

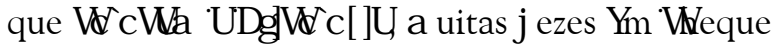
com questionamentos como "porque a terapia funciona?". Além disso, é provável que essa nova abordagem da psicopatologia estimule os psicólogos a embasarem suas técnicas visando uma mudança cerebral específica em termos de neurogênese, sinaptogênese e plasticidade a partir dos achados das neurociências cognitivas.

A nova visão do psicólogo como um "profissional do corpo" (Gonçalves \& Nogueira, 1999) gerou uma outra consequência para a prática em saúde mental. Em 1996 a APA publicou os modelos de treinamento e de legislação para a política "RxP" [1] , que tem como objetivo ampliar a atuação dos psicólogos clínicos em prescrever medicações psiquiátricas (Heiby, DeLeon, \& Anderson, 2004). Essa é uma consequência mais indireta e distante dos achados científicos demonstrados nesse trabalho, mas considerada de relevância nessa discussãopois, em nossa concepção, esse movimento só foi possível nesse momento específico da história da ciência, e não em outro, justamente em consequência dos novos conhecimentos trazidos pelas investigações das neurociências cognitivas sobre a psicopatologia e a psicoterapia. O argumento científico utilizado pelos defensores dessa atividade é que a psicoterapia, assim como a psicofarmacologia, intervêm no cérebro, algumas vezes por vias diferentes, algumas vezes pelas mesmas vias. 
Os psicólogos estão igualmente divididos nas opiniões sobre adotar ou não essa política (Robiner et al., 2003; Walters, 2001). Os principais argumentos contra a prescrição dos psicólogos são de natureza de treinamento (Robiner et al., 2003). Os principais argumentos a favor da prescrição dos psicólogos são de natureza política e em prol da maior eficácia do sistema de saúde americano, uma vez que há uma necessidade muito grande de profissionais que administrem drogas psicoativas em muitas regiões do país. A discussão está a avançar e estudos piloto no Estado de Novo México poderão oferecer mais evidências para avaliar a eficácia dessa proposta (Heiby et al., 2004).

Essa proposta é coerente com um movimento muito maior dentro da ciência, dentro do qual a psicologia está em parte inserida. Entretanto, apesar dessas evidências e novas perspectivas, a impressão é de que estamos apenas começando e a psicologia ainda está longe de se render ao "modelo médico" de saúde mental. Em um estudo de opinião (Levine \& Schmelkin, 2006) psicólogos foram questionados sobre qual a etiologia de alguns dos principais diagnósticos do DSM. Os resultados indicaram que a posição dos psicólogos ainda é bastante moderada entre as causas sociais e as biológicas. Isso não valeu para aqueles que seguem uma orientação cognitivocomportamental, que tendem a apreciar mais os avanços da perspectiva biológica. Os autores concluíram que, de uma maneira geral, " os psicólogos ainda estão longe de abandonar suas raízes filosóficas e teóricas" (p. 208).

\section{Agradecimentos}

Os autores gostariam de agradecer o Professor

Dr\$aulo Araújo pela leitura atenta manuscrito.

\section{Referências}

Araújo, S. de F. (2003). Psicologia e Neurociência: Uma Avaliação da Perspectiva Materialista no
Estudo dos Fenômenos Mentais (1o ed). Juiz de Fora, MG: Editora UFJF.

Baddeley, A., Bueno, O., Cahill, L., Fuster, J. M., Izquierdo, I., McGaugh, J. L., ... Da Cunha, C. (2000). The brain decade in debate: I. Neurobiology of learning and memory. Brazilian Journal of Medical and Biological Research, 33(9), 993-1002. http://doi.org/1 0.1590/S0100-879X2000000900002

Churchland, P. M. (1993). Matter and Consciousness: a contemporary introduction to the philosophy of mind. London: MIT Press.

Derrogatis, L. R. (1977). Symptoms Checklist-90 (SCL-90): Administration, scoring and procedures manual - I for the (revised) version. John Hopkins University School of Medicine, Clinical Psychometrics Research Unit: Baltimore.

Domes, G., Heinrichs, M., Michel, A., Berger, C., \& Herpertz, S. C. (2007). Oxytocin Improves "Mind-Reading" in Humans. Biological Psychiatry, 61 (6), 731-733. http:/ /doi.org/10.1016/j.biopsych.2006.07.015

Eisch, A. J., \& Nestler, E. J. (2002). To be or not to be: adult neurogenesis and psychiatry. Clinical Neuroscience Research, 2(1-2), 93108. http://doi.org/10.1016/S1566-2772(02 )00011-7

Fuchs, E., Uno, H., \& Flügge, G. (1995). Chronic psychosocial stress induces morphological alterations in hippocampal pyramidal neurons of the tree shrew. Brain Research, 673(2), 275-282.

Goldapple, K., Segal, Z., Garson, C., Lau, M., Bieling, P., Kennedy, S., \& Mayberg, H. (2004). Modulation of cortical-limbic pathways in major depression: Treatmentspecific effects of cognitive behavior therapy. Archives of General Psychiatry, 61 (1), 34-41. http://doi.org/10.1001/archp syc.61.1.34

Gonçalves, Ó. F., Cerqueira, J., Guimarães, C., Belpalme, J., Amorim, L., Peixoto, M., \& Sousa, N. (2006). Neurociencias y psicoterapia: retorno a lo básico. Revista de psicoterapia, 16(61), 65-74.

Gonçalves, Ó. F., \& Nogueira, R. de S. S. (1999). Anatomias da psicoterapia: A 
cabeça também é corpo. Revista Portuguesa de Psicossomática, 1(1), 63-77.

Heiby, E. M., DeLeon, P. H., \& Anderson, T. (2004). A Debate on Prescription Privileges for Psychologists. Professional Psychology: Research and Practice, 35 (4), 336-344.

Jeong, Y.-J., Hong, S.-C., Lee, M. S., Park, M.-C., Kim, Y.-K., \& Suh, C.-M. (2005). Dance movement therapy improves emotional responses and modulates neurohormones in adolescents with mild depression. International Journal of Neuroscience, 115(12), 1711-1720.

Jockers-Scherübl, M. C., Zubraegel, D., Baer, T., Linden, M., Danker-Hopfe, H., ... Hellweg, R. (2007). Nerve growth factor serum concentrations rise after successful cognitive-behavioural therapy of generalized anxiety disorder. Progress in Neuro-Psychopharmacology and Biological Psychiatry, 31(1), 200-204. http://doi.org/1 0.1016/j.pnpbp.2006.09.006

Johnston, M. V. (2004). Clinical disorders of brain plasticity. Brain and Development, 26(2), 73-80. http://doi.org/10.1016/S0387 $-7604(03) 00102-5$

Kandel, E. R. (1998). A new intellectual framework for psychiatry. The American Journal of Psychiatry, 155(4), 457-469. http ://doi.org/10.1176/ajp.155.4.457

Kazdin, A. E. (2007). Mediators and Mechanisms of Change in Psychotherapy Research. Annual Review of Clinical Psychology, 3(1), 1-27. http://doi.org/10.1146/annurev.clinp sy.3.022806.091432

Koch, J. M., Kell, S., Hinze-Selch, D., \& Aldenhoff, J. B. (2002). Changes in CREBphosphorylation during recovery from major depression. Journal of Psychiatric Research, 36(6), 369-375. http://doi.org/10 $.1016 / \mathrm{S} 0022-3956(02) 00056-0$

Kumari, V. (2006). Do psychotherapies produce neurobiological effects? Acta Neuropsychiatrica, 18(2), 61-70. http://doi. org/10.1111/j.1601-5215.2006.00127.x

Levine, E. S., \& Schmelkin, L. P. (2006). The move to prescribe: A change in paradigm? Professional Psychology: Research and Practice, 37(2), 205-209. http://doi.org /10.1037/0735-7028.37.2.205

McGinn, C. (1996). The character of the mind: An Introduction to the Philosophy of Mind. New York: Oxford University Press.

Meaney, M. J., \& Szyf, M. (2005). Maternal care as a model for experiencedependent chromatin plasticity? Trends in Neurosciences, 28(9), 456-463. http://doi.o $\mathrm{rg} / 10.1016 / \mathrm{j}$.tins.2005.07.006

Nakatani, E., Nakgawa, A., Ohara, Y., Goto, S., Uozumi, N., ... Yamagami, T. (2003). Effects of behavior therapy on regional cerebral blood flow in obsessivecompulsive disorder. Psychiatry Research: Neuroimaging, 124(2), 113-120. http://doi. org/10.1016/S0925-4927(03)00069-6

Ochsner, K. N., Bunge, S. A., Gross, J. J., \& Gabrieli, J. D. E. (2002). Rethinking Feelings: An fMRI Study of the Cognitive Regulation of Emotion. Journal of Cognitive Neuroscience, 14(8), 1215-1229. http://doi. org/10.1162/089892902760807212

Robiner, W. N., Bearman, D. L., Berman, M., Grove, W. M., Colón, E., ... Tanenbaum, R. L. (2003). Prescriptive Authority for Psychologists: Despite Deficits in Education and Knowledge? Journal of Clinical Psychology in Medical Settings, 10(3), 211-221. http://doi.org/10.1023/A:102541 9114038

Roffman, J. L., Marci, C. D., Glick, D. M., Dougherty, D. D., \& Rauch, S. L. (2005). Neuroimaging and the functional neuroanatomy of psychotherapy. Psychological Medicine, 35(10), 13851398. http://doi.org/10.1017/S0033291705 005064

Scorza, F. A., Guerra, A. de B. G., Cavalheiro, E. A., \& Calil, H. M. (2005). Neurogênese e depressão: etiologia ou nova ilusão? Neurogenesis and depression: etiology or new ilusion? Revista Brasileira de Psiquiatria, 27(3), 249-53.

Sheline, Y. I., Wang, P. W., Gado, M. H., Csernansky, J. G., \& Vannier, M. W. (1996). Hippocampal atrophy in recurrent 
major depression. Proceedings of the National Academy of Sciences, 93(9), 3908-3913.

Walters, G. D. (2001). A meta-analysis of opinion data on the prescription privilege debate. Canadian Psychology/Psychologie canadienne, 42 (2), 119-125. http://doi.org/10.1037/h00 86886

\section{Notas}

\section{* Reflection Article.}

[1] "Rx" é o símbolo usado na medicina para a prescrição de medicamentos. $\mathrm{O}$ "P" no final indica a autoridade de prescrição do psicólogo. 